\section{(6) OPEN ACCESS}

\title{
Effectiveness and safety of sofosbuvir plus ribavirin for the treatment of HCV genotype 2 infection: results of the real-world, clinical practice HCV-TARGET study
}

\author{
Tania M Welzel, ${ }^{1}$ David R Nelson, ${ }^{2}$ Giuseppe Morelli, ${ }^{2}$ Adrian Di Bisceglie, ${ }^{3}$ \\ Rajender K Reddy, ${ }^{4}$ Alexander Kuo, ${ }^{5}$ Joseph K Lim, ${ }^{6}$ Jama Darling, ${ }^{7}$ Paul Pockros, ${ }^{8}$ \\ Joseph S Galati, ${ }^{9}$ Lynn M Frazier, ${ }^{10}$ Saleh Alqahtani, ${ }^{11}$ Mark S Sulkowski, ${ }^{11}$ \\ Monika Vainorius, ${ }^{7}$ Lucy Akushevich, ${ }^{7}$ Michael W Fried, ${ }^{7}$ Stefan Zeuzem, ${ }^{1}$ \\ for the HCV-TARGET Study Group
}

Additional material is published online only. To view please visit the journal online (http://dx.doi.org/10.1136/ gutjnl-2016-311609).

For numbered affiliations see end of article.

Correspondence to Dr Tania Mara Welzel, JW Goethe University Hospital, Department of Medicine 1, Theodor-Stern-Kai 7, Frankfurt am Main 60596, Germany; tania.welzel@kgu.de

Received 6 February 2016 Revised 1 June 2016 Accepted 4 June 2016 Published Online First 12 July 2016

CrossMark

To cite: Welzel TM,

Nelson DR, Morelli $G$, et al.

Gut 2017;66:1844-1852.

\section{ABSTRACT}

Objective Due to a high efficacy in clinical trials, sofosbuvir (SOF) and ribavirin (RBV) for 12 or 16 weeks is recommended for treatment of patients with HCV genotype (GT) 2 infection. We investigated safety and effectiveness of these regimens for GT2 in HCV-TARGET participants.

Design HCV-TARGET, an international, prospective observational study evaluates clinical practice data on novel antiviral therapies at 44 academic and 17 community medical centres in North America and Europe. Clinical data were centrally abstracted from medical records. Selection of treatment regimen and duration was the investigator's choice. The primary efficacy outcome was sustained virological response 12 weeks after therapy (SVR12).

Results Between December 2013 and April 2015, 321 patients completed 12 weeks ( $n=283$ ) or 16 weeks $(n=38)$ of treatment with SOF and RBV. Prior treatment experience and cirrhosis was more frequent among patients in the 16-week regimen compared with 12 weeks ( $52.6 \%$ vs $27.6 \%$ and $63.2 \%$ vs $21.9 \%$, respectively). Overall, SVR12 was $88.2 \%$. The SVR12 in patients without cirrhosis was $91.0 \%$ and $92.9 \%$ for 12 or 16 weeks of therapy, respectively. In patients with cirrhosis treated for 12 or 16 weeks, SVR12 was $79.0 \%$ and $83 \%$. In the multivariate analysis, liver cirrhosis, lower serum albumin and RBV dose at baseline were significantly associated with SVR12. Common adverse events (AEs) included fatigue, anaemia, nausea, headache, insomnia, rash and flu-like symptoms. Discontinuation due to AEs occurred in $2.8 \%$.

Conclusions In this clinical practice setting, SOF and RBV was safe and effective for treatment of patients with HCV GT2 infection.

Trial registration number NCT01474811.

\section{INTRODUCTION}

Chronic infection with $\mathrm{HCV}$ is a major cause of end-stage liver disease and hepatocellular

\section{Significance of this study}

What is already known on this subject?

- Due to a high efficacy in clinical trials, the all-oral combination of sofosbuvir (SOF) and ribavirin (RBV) for 12 weeks is currently considered standard of care in patients with HCV genotype 2 (GT2) infection.

- Based on limited data from clinical trials, treatment extension to 16 weeks is recommended by international HCV treatment guidelines for patients with negative predictors of treatment response, including patients with liver cirrhosis.

- Information on the effectiveness and safety of SOF and RBV for treatment of HCV GT2 in real-world, clinical practice is limited.

\section{What are the new findings?}

- Sustained virological response 12 weeks after therapy (SVR12) rates as observed in HCV-TARGET, a prospective, international, multicentre 'real-word' study that included a larger number of patients with HCV GT2 infection than any pivotal study generally confirm the effectiveness and safety of SOF and RBV for treatment of GT2.

- While response rates in patients without cirrhosis were high and comparable with those reported in clinical trials, liver cirrhosis, lower serum albumin levels and RBV dose at baseline were associated with lower SVR12 rates.

- Only a small proportion of patients with cirrhosis $(27 \%)$ were treated for 16 weeks, and response rates were similar to those treated for 12 weeks. Larger, randomised and adequately powered trials would be necessary to assess the benefit of treatment extension to 16 weeks in cirrhotics. 
Significance of this study

\section{How might it impact on clinical practice in the foreseeable future? \\ - This large, international cohort study confirms the effectiveness and safety of the all-oral combination of SOF and RBV for treatment of HCV GT2. \\ - The results from recent phase III studies demonstrating that SOF and velpatasvir is superior to SOF plus RBV must be confirmed in real-world studies.}

carcinoma (HCC). ${ }^{1}{ }^{2}$ Sustained virological response 12 weeks after therapy (SVR12) is associated with a decreased risk for liver-related morbidity and mortality. ${ }^{3} 4$

Among the seven HCV genotypes (GTs) isolated until now, HCV genotype 2 (GT2) is the third most common GT with an estimated 16.5 million GT2-infected persons worldwide. Relative proportions of HCV GT2 infections range from 10.8\% and $12 \%$ in Western Europe and North America to higher prevalences in Central Latin America (19.3\%), Western Sub-Saharan Africa (23.0\%) and Asia Pacific (24.5\%). ${ }^{5} 6$ Compared with other HCV GTs such as GT1, GT3 and GT4, historic therapy with pegylated interferon (PegIFN) and ribavirin (RBV) for 24 weeks yielded favourable response rates in GT2-infected patients. ${ }^{7}$ However, the poor tolerability limited the clinical use of PegIFN-based therapies, in particular in patients with liver cirrhosis.

The availability of all-oral, direct-acting antiviral (DAA) drug combinations with high efficacy, favourable side-effect profile and easy applicability facilitated treatment of HCV infection in clinical practice. ${ }^{8} 9$ Sofosbuvir (SOF), a first in-class nucleotide NS5B polymerase inhibitor with a high barrier to resistance, was approved for interferon-free treatment of HCV infection in the USA and Europe in early 2014. ${ }^{10}$

For HCV GT2, the efficacy and safety of SOF in combination with weight-based RBV for 12 weeks was investigated in several pivotal phase III trials: FISSION, POSITRON and VALENCE. ${ }^{11-13}$ In these trials, 94\% of patients without cirrhosis and $90 \%$ of patients with cirrhosis achieved a SVR12. Another phase III study (FUSION) that compared SOF and RBV for 12 or 16 weeks indicated that patients with liver cirrhosis may benefit from extended treatment duration. ${ }^{12}$ This study, however, only included a small number of patients with cirrhosis; SVR12 rates were $60 \%$ and $78 \%$ in patients treated for 12 and 16 weeks, respectively. Based on these results, SOF and RBV for 12 weeks is currently recommended for most patients with HCV GT2 infection by US, Canadian and European guidelines, although extension to 16 weeks may be considered for patients with cirrhosis. ${ }^{14-17}$

Information derived from usual clinical practice on treatment of HCV GT2 with SOF and RBV is limited ${ }^{18} 19$ and outcomes in selected study populations may differ from those observed in clinical practice. The aim of this study was to investigate the effectiveness and tolerability of SOF and RBV for treatment of HCV GT2 in HCV-TARGET, an international, prospective observational academic consortium study, designed to evaluate information on novel DAA therapies used in routine patient care. $^{20}$

\section{MATERIALS AND METHODS}

\section{Patients}

HCV-TARGET is a prospective, longitudinal, observational study conducted at 44 academic and 17 community medical centres in North America (57 centres) and Europe (4 centres). The registry enrolls adult patients with $\mathrm{HCV}$ ( $\geq 18$ years), who initiated HCV treatment with DAA agents according to the recommendation of the site investigator. For participation, patients had to provide informed consent within 4 weeks after treatment initiation. This study includes data from patients with HCV GT2 infection sequentially enrolled in HCV-TARGET between 8 December 2013 and 15 April 2015. Patients completed treatment with SOF and weight-based RBV for a duration of either 12 or 16 weeks (with a duration window of \pm 2 weeks to account for practice variability). Patients were excluded from this analysis if they had undergone liver transplantation or had previously been treated with a prior DAA.

\section{Treatments}

SOF was prescribed at an oral standard dose of $400 \mathrm{mg}$ once daily. Selection of the appropriate RBV dose and determination of the treatment duration were the physician's choice. According to the label, the recommended RBV dose is $1000 \mathrm{mg} /$ day $(\leq 75 \mathrm{~kg}$ ) or 1200 (>75 kg) mg/day, divided into two daily doses.

\section{Data collection}

Demographic and clinical information, laboratory tests and technical exams were performed according to the site's standards. Data were collected within a centralised database using standardised source data abstraction as described previously. ${ }^{21}$ The database was managed using Research Electronic Data Capture (REDCap) tools hosted at the University of North Carolina, Chapel Hill. ${ }^{22}$ To optimise data quality, a trained Centralized Chart Data Abstraction Team at the Clinical Coordinating Center at the University of Florida was established to enter de-identified copies of source data, that is, clinical records that were provided by participating sites. Special effort was made to explore reasons for premature discontinuations and to investigate the reasons for missing data and to minimise loss to follow-up. Independent data monitors reviewed data entries for completeness and accuracy.

Cirrhosis status was assessed by availability of a liver biopsy or a combination of several clinical and/or imaging criteria previously described. ${ }^{21}$ Briefly, cirrhosis was defined by (1) liver biopsy indicating liver fibrosis stage 4 (Metavir) prior to treatment initiation and (2) liver biopsy indicating stage 3 fibrosis prior to treatment initiation in combination with any of the following clinical parameters: platelets $<140000 / \mu \mathrm{L}$, oesophageal varices on oesophagogastroduodenoscopy, cirrhosis and/or portal hypertension and/or of ascites by imaging methods, FibroSure (or equivalent) test, vibration-controlled transient elastography (VCTE) or equivalent compatible with fibrosis stage 4. If a liver biopsy was not available, cirrhosis was defined by evidence for two of the following variables: platelet count $<140000 / \mu \mathrm{L}$, evidence for oesophageal varices on oesophagogastroduodenoscopy, cirrhosis and/or portal hypertension and/ or ascites as diagnosed by imaging studies, FibroSure or equivalent test, VCTE or equivalent compatible with stage 4 fibrosis. Decompensated cirrhosis was defined as the presence of current or past ascites, hepatic encephalopathy, spontaneous bacterial peritonitis, hepatic hydrothorax, variceal haemorrhage or concomitant medications with a specific indication for the beforementioned conditions. 


\section{Efficacy and safety endpoints}

HCV RNA was determined according to local standard molecular tests. Primary efficacy endpoint was SVR12 defined as HCV RNA below the lower limit of quantification or undetectable at least 64 days after treatment was discontinued (allowing for window around usual clinic visits). An analysis was performed of the evaluable population (EP), defined as the group of patients who have completed either 12 -week or 16 -week regimen and have the final treatment outcome available, including those who were lost to post-treatment follow-up (counted as treatment failures). An analysis of the per protocol (PP) population was also performed in the group of patients who completed 12-week or 16-week regimen and have virological outcomes available.

Adverse events (AEs) were recorded in the medical record regularly at patient visits. Anaemia was defined as either anaemia reported as an AE or documented RBV dose modification, application of erythropoietin or transfusion. Serious AEs/suspected unexpected serious adverse reactions were reported by sites by submission of Voluntary Form FDA3500 (MedWatch) to the Clinical Coordinating Center at the University of Florida.

\section{Statistical analysis}

Demographics, baseline laboratory values, outcomes and frequencies of AEs were collected and analysed for the EP according to discrete subpopulations of interest, particularly based on the duration of treatment (12 and 16 weeks). Virological outcomes and associations between sustained virological response (SVR) and baseline patient characteristics, baseline RBV dose (per kg), RBV management during treatment and treatment duration were explored among PP patients.

Multivariable analyses with Firth's penalised estimation of predictors of sustained virological response were run for the SOFRBV patients and measures of association between selected covariates and SVR were estimated with logistic regression with the predictor of interest with 'minimal' covariate adjustment for age and gender. A set of univariable logistic models as well as a set of multivariable logistic models with the predictor of interest, age, gender and prior antiviral treatment experience and cirrhotic status in the model were performed for sensitivity analysis (not shown). The set of predictor variables were selected a priori based on a consensus of clinical expertise and included the well-established baseline covariates associated with SVR: cirrhosis status, gender, age, race, model for end-stage liver disease (MELD), albumin (g/dL), platelet count $(1000 / \mu \mathrm{L})$, creatinine clearance, total bilirubin $(\mathrm{mg} / \mathrm{dL})$, haemoglobin $(\mathrm{g} / \mathrm{dL})$, international normalised ratio, RBV dose at baseline $(\mathrm{mg} / \mathrm{kg})$ and $\mathrm{RBV}$ dose reduction. The results are presented with an OR and 95\% CIs. Patients who prematurely discontinued due to any reason (there are no discontinuations due to lack of efficacy) or were lost to follow-up are excluded from the multivariable analyses. Analyses were performed using SAS software V.9.4 (SAS Institute, Cary, North Carolina, USA).

\section{Study oversight}

All patients provided written informed consent prior to their participation. The study was conducted in accordance with the Declaration of Helsinki and Good Clinical Practice guidelines. All authors had access to the data and approved the final version of the manuscript.

\section{RESULTS \\ Patient population}

Between December 2013 and April 2015, 361 patients with chronic HCV GT2 infection with no history of liver transplantation or prior DAA therapy initiated treatment with SOF and RBV. Of those, 24 discontinued early for the following reasons: AEs 10 (2.8\%), lost to on-treatment follow-up 9 (2.5\%), non-compliance $3(1 \%)$ and $2(<1 \%)$ due to other reasons. Of 337 patients who completed treatment, 10 patients who received unconventional treatment durations (neither 12 nor 16 weeks) and 6 patients who still continued in posttreatment follow-up were excluded from our analyses. The EP thus includes 321 patients. Of those, 307 had completed treatment (for either 12 or 16 weeks) and ended post-treatment follow-up, and 14 patients were lost to post-treatment follow-up (figure 1).

Demographic and clinical characteristics of the study participants are shown in table 1 . The median age of the patients was 59 years. The majority was white $(276 / 321 ; 86.0 \%)$ and male (196/321; 61.1\%). $30.5 \%$ of the patients were experienced to prior treatment with PegIFN and RBV. Cirrhosis was present in 86 of $321(26.8 \%)$ patients. Of these, $36(41.9 \%)$ had evidence of current or prior decompensation. Compared with patients treated for 12 weeks, the proportion of patients with prior treatment experience and cirrhosis was higher in the group who received 16 weeks of treatment. Among cirrhotics, a higher percentage in the 16 -week group had received prior therapy $(17 / 24$ $(70.8 \%)$ vs $30 / 62(48.4 \%))$ and had MELD scores of $\geq 10$ (57.9\% vs $39.5 \%)$; also, the mean platelet counts were lower in patients with cirrhosis who received the extended treatment duration (77 vs $\left.105.5 \times 10^{3} / \mu \mathrm{L}\right)$.

\section{Efficacy}

The SVR12 rates for the EP and for the PP population, stratified by prior treatment (treatment naïve, experienced) and cirrhosis status are shown in table 2. Overall, 283 of 321 patients (88.2\%) achieved a SVR12 in the EP, while 283 of 307 (92.2\%) in the PP population had a SVR12 (table 2). In the EP analysis, $283(88.2 \%)$ and $38(11.8 \%)$ of 321 patients received SOF and RBV for 12 or 16 weeks, respectively. The PP population included $271(88.3 \%)$ and $36(11.7 \%)$ patients treated for 12 and 16 weeks, respectively. Among GT2-infected patients treated for 12 weeks, the overall SVR12 rate (EP) was $88.3 \%$ $(250 / 283)$ and $86.8 \%(33 / 38)$ in those treated for 16 weeks. Corresponding SVR12 rates in the PP population were $92.3 \%$ (250/271) and 91.7\% (33/36), respectively. In patients without cirrhosis, SVR12 (EP) was 91.0\% (201/221) in patients who received 12 weeks of SOF and RBV and 92.9\% (13/14) among patients treated for 16 weeks. SVR12 rates (EP) in patients with cirrhosis treated for 12 or 16 weeks were $79.0 \%(49 / 62)$ and $83.3 \%$ (20/24), respectively. In the PP population, $93.9 \%$ $(201 / 214)$ and $100 \%(13 / 13)$ of patients without cirrhosis and $86.0 \%(49 / 57)$ and $87.0 \%(20 / 23)$ of patients who received 12 or 16 weeks of treatment achieved a SVR12. SVR12 rates for the EP and PP population, further stratified by prior treatment status (naïve vs experienced) are shown in table 2. SVR12 rates (PP) were similar in US versus European patients; 9 of 10 (90\%) (PP) of the patients treated in Europe and 274 of 311 $(88.1 \%)$ of the US patients achieved a virological response.

We also investigated the impact of baseline haemoglobin, baseline RBV dose and RBV dose reduction on SVR12. Figure 2 shows the probability of achieving a SVR12 by RBV dose at baseline $(\mathrm{mg} / \mathrm{kg})$. Lower baseline RBV doses were associated with a lower probability of SVR12 and optimal chances for SVR12 are predicted for baseline RBV doses of $15 \mathrm{mg} / \mathrm{kg}$ bodyweight (BW) and higher. In patients with RBV dose reductions, PP SVR12 rates were 94.0\% (78/83) compared with $91.5 \%$ $(205 / 224)$ in patients without RBV dose reductions. In patients 


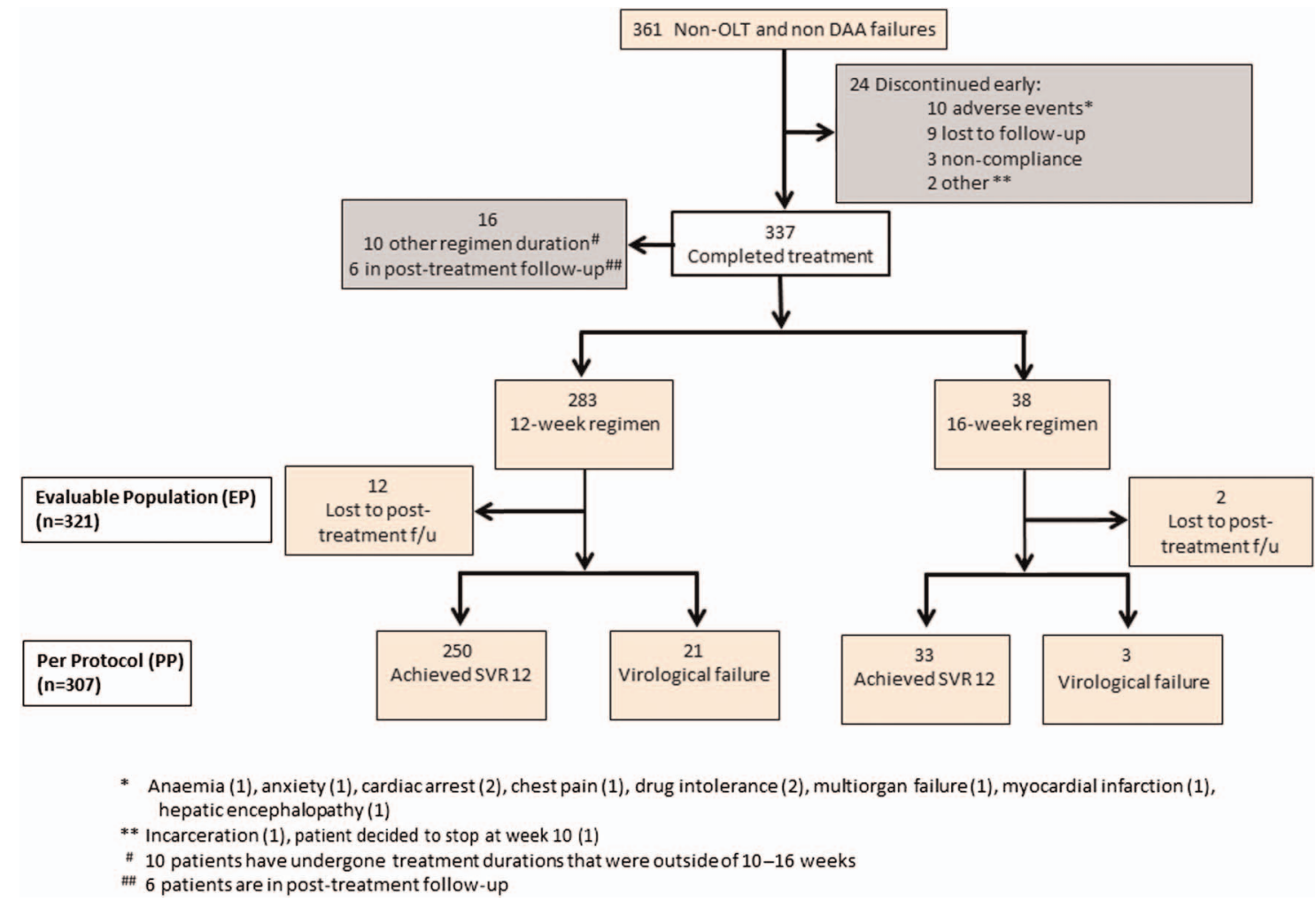

Figure 1 Patient disposition. DAA, direct-acting antiviral; f/u, follow-up; OLT, orthotopic liver translantation; SVR12, sustained virological response 12 weeks after therapy.

with cirrhosis, the corresponding SVR12 rates were 92.9\% (28/28) and $82.7 \%(43 / 52)$, respectively.

Of the 38 patients who did not achieve a SVR12, 24 had a virological failure and 14 were lost to post-treatment follow-up. Relapse was the most common reason for virological failure (21/24, 87.5\%); viral breakthrough was reported in two and non-response in one patient (table 2).

In the multivariable minimally adjusted regression analysis, the absence of cirrhosis and higher serum albumin demonstrated a significant association with SVR12 (OR 2.4, 95\% CI 1.02 to 5.54; and OR 3.2, 95\% CI 1.51 to 6.9, respectively) (figure 3). Baseline haemoglobin and RBV dose reductions did not impact SVR12 rates. However, there was a small, although statistically significant $(p=0.024)$ impact of higher baseline RBV dose (mg/kg) and SVR12 (OR 1.2, 95\% CI 1.02 to 1.33 ). Sensitivity analysis was performed employing univariable analysis as well as multivariable analysis adjusting for cirrhosis status and prior treatment experience in addition to age and sex (data not shown). Both methods support the findings of the minimally adjusted model. There were no significant changes in MELD score, platelet count, total bilirubin and albumin in patients with cirrhosis who achieved SVR12 compared with those with virological failure within the study's treatment and follow-up period (see online supplemental figure S1). The change was measured between the values recorded prior to treatment start and values recorded 10 weeks or later (latest available) after treatment end.

\section{Safety}

Only 10 of $361(2.8 \%)$ patients discontinued treatment prematurely due to an AE. Of those, eight patients had liver cirrhosis. The reasons for drug discontinuation, each reported in one of the patients, included anaemia, anxiety, chest pain, hepatic encephalopathy, multiorgan failure and cardiac arrest, while two patients each were reported to have decreased drug tolerance and myocardial infarction.

Six patients (1.9\%), all patients with cirrhosis, who started treatment with SOF and RBV died. The reported cause of death was multiorgan failure in three patients, cardiac arrest in two patients and hepatic encephalopathy in one patient. All deaths were considered unrelated to the antiviral medication by the treating physicians. Of the patients with multiorgan failure, one had a myocardial infarction; another patient discontinued SOF and RBV on treatment day 2 due to multiorgan failure and another patient had a multiorgan failure 27 days after the last intake of SOF and RBV. Of the patients with cardiac arrest, one patient had a car accident with cardiac arrest reported as a cause of death the subsequent day; another patient had a HCC at enrollment in HCVTARGET and cardiac arrest was reported as a cause of death on treatment day 98 . None of the two patients with reported cardiac arrest had received amiodarone or calcium channel blockers. Reported heart disease-related medications included nadolol and benazepril in one, and nadolol, spironolactone and furosemide in the other patient. The patient with hepatic encephalopathy had a history of hepatic decompensation at initiation of treatment and expired on treatment day 98 . The event was not considered to be treatment related by the treating physician.

AEs were reported in 268/321 (83.5\%) patients of the EP (table 3). Most common AEs, reported in $\geq 10 \%$ of the patients, included fatigue, anaemia, nausea, headache, insomnia, rash, influenza-like illness and dyspnoea. Anaemia was reported in 68 patients $(21.2 \%)$ and was more frequent in patients who were treated for 16 weeks, compared with those who received 12 weeks of therapy (36.8\% vs $19.1 \%$, respectively), possibly also attributable to the higher proportion of patients with liver 
Table 1 Baseline demographic and clinical characteristics among patients with genotype 2 (GT2) infection who completed therapy with sofosbuvir (SOF) and ribavirin (RBV)

\begin{tabular}{|c|c|c|c|c|c|c|}
\hline \multirow[b]{2}{*}{ Characteristic } & \multicolumn{3}{|c|}{ Overall cohort } & \multicolumn{3}{|c|}{ Cirrhotics } \\
\hline & $\begin{array}{l}\text { SOF plus RBV for } \\
12 \text { weeks }(n=283)\end{array}$ & $\begin{array}{l}\text { SOF plus RBV for } \\
16 \text { weeks }(n=38)\end{array}$ & Total $(n=321)$ & $\begin{array}{l}\text { SOF plus RBV for } \\
12 \text { weeks }(n=62)\end{array}$ & $\begin{array}{l}\text { SOF plus RBV for } \\
16 \text { weeks }(n=24)\end{array}$ & Total $(n=86)$ \\
\hline Median age (range), years & $59.0(21-80)$ & $62.5(30-78)$ & $59.0(21-80)$ & $60(40-78)$ & $62(46-78)$ & $60.5(40-78)$ \\
\hline $18-39$ & $22(7.8 \%)$ & $2(5.3 \%)$ & $24(7.5 \%)$ & $0(0.0 \%)$ & $0(0.0 \%)$ & $0(0.0 \%)$ \\
\hline $40-64$ & $202(71.4 \%)$ & $22(57.9 \%)$ & $224(69.8 \%)$ & $49(79.0 \%)$ & $16(66.7 \%)$ & $65(75.6 \%)$ \\
\hline $65+$ & $59(20.8 \%)$ & $14(36.8 \%)$ & $73(22.7 \%)$ & $13(21 \%)$ & $8(33.3 \%)$ & $21(24.4 \%)$ \\
\hline Male sex, n (\%) & $174(61.5 \%)$ & $22(57.9 \%)$ & $196(61.1 \%)$ & $40(64.5 \%)$ & $14(58.3 \%)$ & $54(62.8 \%)$ \\
\hline \multicolumn{7}{|l|}{ Race, n (\%) } \\
\hline White & $243(85.9 \%)$ & $33(86.8 \%)$ & $276(86.0 \%)$ & $55(88.7 \%)$ & $22(91.7 \%)$ & 77 (89.5\%) \\
\hline Black & $16(5.7 \%)$ & $2(5.3 \%)$ & $18(5.6 \%)$ & $2(3.2 \%)$ & $0(0.0 \%)$ & $2(2.3 \%)$ \\
\hline Other & $24(8.5 \%)$ & $3(7.9 \%)$ & $27(8.4 \%)$ & $5(8.1 \%)$ & $2(8.3 \%)$ & $7(8.1 \%)$ \\
\hline Hispanic ethnicity, $\mathrm{n}(\%)$ & $22(7.8 \%)$ & $5(13.2 \%)$ & $27(8.4 \%)$ & $7(11.3 \%)$ & $3(12.5 \%)$ & $10(11.6 \%)$ \\
\hline \multicolumn{7}{|l|}{ Prior HCV treatment, $\mathrm{n}(\%)$} \\
\hline Naive & $205(72.4 \%)$ & $18(47.4 \%)$ & $223(69.5 \%)$ & $32(51.6 \%)$ & $7(29.2 \%)$ & $39(45.3 \%)$ \\
\hline Experienced & $78(27.6 \%)$ & $20(52.6 \%)$ & $98(30.5 \%)$ & $30(48.4 \%)$ & $17(70.8 \%)$ & $47(54.7 \%)$ \\
\hline Cirrhosis, n (\%) & $62(21.9 \%)$ & $24(63.2 \%)$ & $86(26.8 \%)$ & $62 / 62(100 \%)$ & $24 / 24(100 \%)$ & $86 / 86(100 \%)$ \\
\hline $\begin{array}{l}\text { History of hepatic decompensation, } \\
\mathrm{n}(\%)\end{array}$ & $28(9.9 \%)$ & $12(31.6 \%)$ & $40(12.5 \%)$ & $25(40.3 \%)$ & $11(45.8 \%)$ & $36(41.9 \%)$ \\
\hline MELD $\geq 10$ (among cirrhotics)* & $17 / 43(39.5 \%)$ & $11 / 19(57.9 \%)$ & $28 / 62(45.2 \%)$ & $17 / 43(39.5 \%)$ & $11 / 19(57.9 \%)$ & $28 / 62(45.2 \%)$ \\
\hline \multicolumn{7}{|l|}{ HCV GT2 subtype } \\
\hline $2 a$ & $18(6.4 \%)$ & $3(7.9 \%)$ & $21(6.5 \%)$ & $3(4.8 \%)$ & $2(8.3 \%)$ & $5(5.8 \%)$ \\
\hline $2 b$ & $125(44.2 \%)$ & $22(57.9 \%)$ & $147(45.8 \%)$ & $31(50.0 \%)$ & $13(54.2 \%)$ & $44(51.2 \%)$ \\
\hline $2 c$ & $2(0.7 \%)$ & $0(0.0 \%)$ & $2(0.6 \%)$ & $0(0.0 \%)$ & $0(0.0 \%)$ & $0(0.0 \%)$ \\
\hline $2 d$ & $1(0.4 \%)$ & $0(0.0 \%)$ & $1(0.3 \%)$ & $0(0.0 \%)$ & $0(0.0 \%)$ & $0(0.0 \%)$ \\
\hline Not reported & $137(48.4 \%)$ & $13(34.2 \%)$ & $150(46.7 \%)$ & $28(41.2 \%)$ & $9(37.5 \%)$ & $37(43 \%)$ \\
\hline EU patients & $11(4.8 \%)$ & $1(2.7 \%)$ & $12(3.8 \%)$ & $4(6.4 \%)$ & $1(4.2 \%)$ & $5(5.8 \%)$ \\
\hline HCV RNA (median), $\log _{10} \mathrm{IU} / \mathrm{mL}$ & 6.3 & 6.4 & 6.3 & 5.9 & 6.0 & 5.9 \\
\hline $\begin{array}{l}\text { Total bilirubin, median (range), } \\
\mathrm{mg} / \mathrm{dL}\end{array}$ & $0.6(0.1-6.2)$ & $0.8(0.3-5.5)$ & $0.6(0.1-6.2)$ & $1.0(0.2-6.2)$ & $1.2(0.3-5.5)$ & $1.0(0.2-6.2)$ \\
\hline Albumin, median (range), g/dL & $4.2(2.2-5.4)$ & $3.8(2.6-4.6)$ & $4.2(2.2-5.4)$ & $3.7(2.2-4.9)$ & $3.7(2.6-4.5)$ & $3.7(2.2-4.9)$ \\
\hline ALT, median (range), IU/L & $53.0(9.0-554.0)$ & $62.5(20.0-428.0)$ & $53.0(9.0-554.0)$ & $49.0(24.0-278.0)$ & $81.0(20.0-428.0)$ & $64.0(20.0-428.0)$ \\
\hline $\begin{array}{l}\text { Platelet count, median (range) } \\
\left(\times 10^{3}\right) / \mu \mathrm{L}\end{array}$ & $189(35.0-407.0)$ & $111(34.0-302.0)$ & $185(34.0-407.0)$ & $105.5(35.0-307.0)$ & 77 (34.0-229.0) & $100(34.0-307.0)$ \\
\hline
\end{tabular}

${ }^{*}$ In patients with available score.

ALT, alanine-aminotransferase; EU, European Union; MELD, model for end-stage liver disease.

cirrhosis in the extended treatment group. Anaemia was managed mostly with RBV dose reductions in $17.8 \%$. Blood transfusions or the use of erythropoietin was documented in $2.5 \%$ and $3.1 \%$ of the patients, respectively.

\section{DISCUSSION}

The results of this large, international observational study that included 321 HCV GT2-infected HCV-TARGET participants demonstrate a high efficacy of SOF and RBV. Overall SVR12 rate was $88.2 \%(283 / 321)$ and the PP SVR12 rate (only including patients who completed 12 -week or 16 -week treatment duration and have virological SVR12 results available) was $92.2 \%$ (283/307). Both treatment durations of SOF in combination with weight-based RBV in this clinical practice setting were well tolerated.

Several randomised phase III trials investigated SOF in combination with RBV for 12 weeks in HCV GT2-infected patients with or without liver cirrhosis. FISSION was a randomised noninferiority trial comparing SOF and RBV for 12 weeks with PegIFN plus RBV for 24 weeks in treatment-naïve GT2-infected patients. Response rates were 97\% (58/61) in patients without cirrhosis and $83 \%(10 / 12)$ in patients with cirrhosis who received SOF and RBV, compared with 81\% (44/54) and 62\% $(8 / 13)$ in respective groups who received PegIFN and RBV. ${ }^{11}$ POSITRON, a placebo-controlled trial, included 109 GT2-infected patients who were ineligible for treatment with PegIFN . Response rates for the 12-week regimen were $92 \%$ $(85 / 92)$ and $94 \%(16 / 17)$ in patients without cirrhosis and patients with cirrhosis, respectively. ${ }^{12}$ The VALENCE trial evaluated SOF with RBV for 12 weeks in GT2-infected patients. High response rates were observed across the different subgroups: 97\% (29/30) and 91\% (30/33) among treatment-naïve and treatment-experienced patients without liver cirrhosis, and $100 \%(2 / 2)$ and $88 \%(7 / 8)$ in treatment-naïve or treatment-experienced patients with cirrhosis. ${ }^{13}$ FUSION was a randomised, double-blinded trial that evaluated 12 or 16 weeks of treatment with SOF and RBV in patients who failed prior interferon-based therapy (relapsers and non-responders). In patients without cirrhosis, response rates observed with 16 weeks did not substantially differ from those obtained with 12 weeks of treatment $(90 \%(26 / 29)$ vs $92 \%(24 / 26))$. In patients with liver cirrhosis, however, the response rate was $78 \%(7 / 9)$ in patients treated for 16 weeks compared with $60 \%$ $(6 / 10)$ in patients treated for 12 weeks. ${ }^{12}$ A recent phase IIIb 


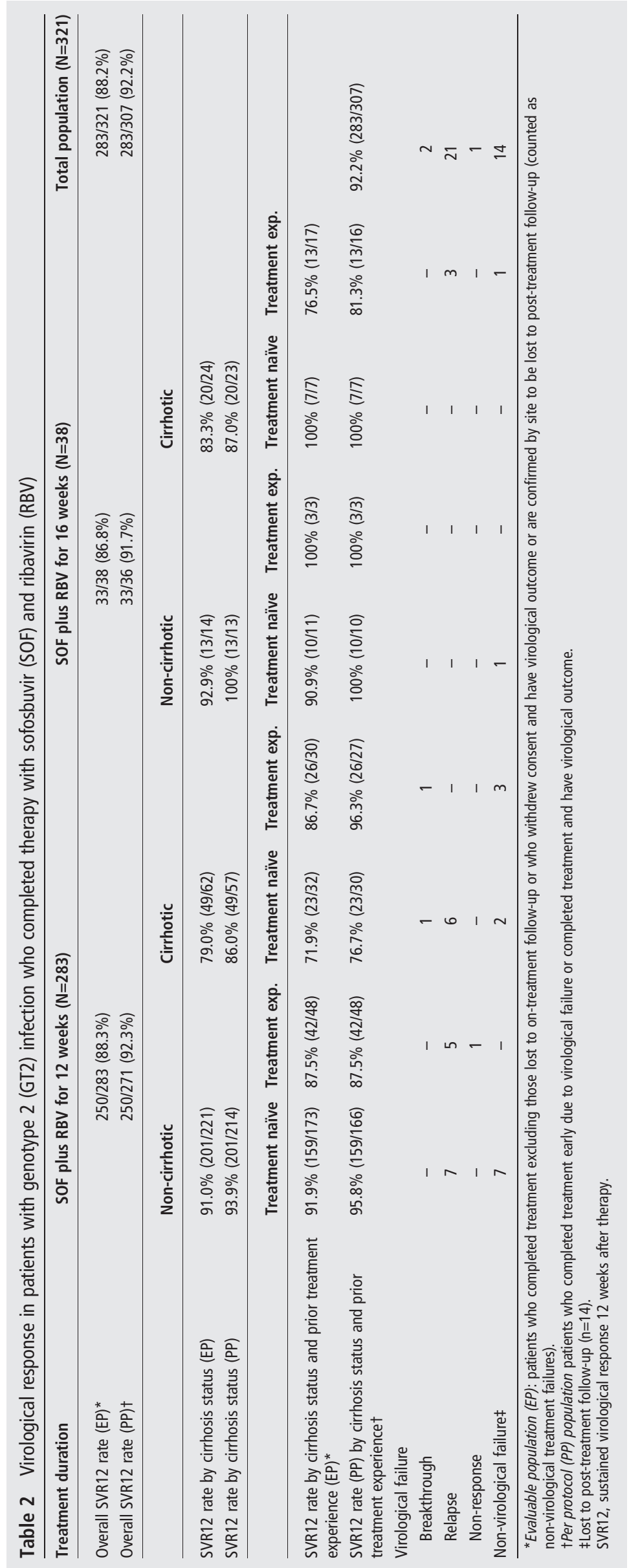




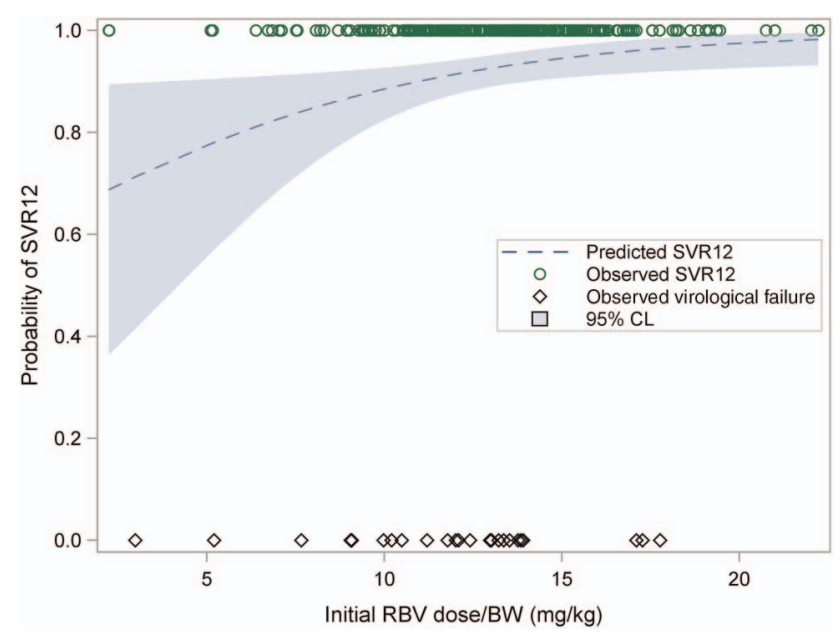

Figure 2 Probability of sustained virological response 12 weeks after therapy (SVR12) by baseline ribavirin (RBV) dose per kg bodyweight (BW; mg/kg). CL, confidence limit.

trial (BOSON) investigated SOF and RBV for 16 or 24 weeks and SOF in combination with PegIFN and RBV in interferon-experienced patients with compensated liver cirrhosis. Response rates were similar across the different treatment groups: $87 \%(15 / 16)$ and $100 \%(17 / 17)$ in patients treated with SOF and RBV for 16 or 24 weeks, and 94\% (15/16) in patients treated with SOF, PegIFN and $\mathrm{RBV}^{23}$ Across these phase III studies, high response rates (90-97\%) were consistently observed in patients with GT2 and without cirrhosis treated with SOF and RBV for 12 weeks. However, numbers of patients with liver cirrhosis enrolled into these trials were small, and response rates in this patient group ranged from $60 \%$ in FUSION to $>87 \%$ in other phase III studies. Also, the comparison and interpretation of different treatment durations (12 vs 16 weeks in FUSION or 16 vs 24 weeks in BOSON) were limited by the small number of patients with cirrhosis included into the respective treatment groups.

Few studies have reported real-world, clinical practice outcomes for SOF and RBV for treatment of HCV GT2. A recent observational study (TRIO Health) evaluated the efficacy of
SOF and RBV for 12 weeks in a real-world population of GT2-infected patients. ${ }^{18}$ In this study, SVR12 rates intent-totreat (ITT) were considerably lower in patients with liver cirrhosis compared with patients without cirrhosis (71\% (32/45) vs $89 \%$ (140/157), respectively). Another observational study investigated the effectiveness of SOF and RBV in GT2-infected US veterans. ${ }^{19}$ Observed response rates among 619 veterans with GT2 infection were generally lower than in pivotal trials and reported SVR rates were $81.6 \%$ and $70.9 \%$ in treatment-naïve and treatment-experienced patients without liver cirrhosis, respectively. In patients with advanced liver disease, SVR12 rates were $58.2 \%$ in those with a FIB-4 $>3.25,53.1 \%$ in those with an aspartate aminotransferase to platelet ratio index (APRI) $>2$ and $44.4 \%$ in patients with a history of decompensated liver disease.

In the present clinical practice study, in PP analysis, SVR12 rates in patients without cirrhosis were comparable with those reported in phase III clinical trials. SVR12 rates in patients without cirrhosis were $93.9 \%$ and $100 \%$ for 12 or 16 weeks of therapy, respectively. In line with other real-world studies, SVR12 rates were lower in patients with liver cirrhosis (86\% and $87 \%$ ). Despite the large number of patients with cirrhosis included into this study, the number of patients with cirrhosis again was not large enough to decide whether treatment prolongation from 12 to 16 weeks has a significant impact. Also, baseline variables among patients with cirrhosis treated for 12 or 16 weeks were not balanced as a higher proportion of patients who received the extended treatment duration had more advanced liver disease. Although the study only included a small number of European GT2-infected patients, SVR12 rates appeared to be similar in US and non-US patients. Baseline haemoglobin levels and RBV dose reductions did not impact SVR12 rates. However, there was a significant impact of lower baseline RBV doses on SVR12 with optimal chances for SVR12 predicted for baseline RBV doses of $15 \mathrm{mg} / \mathrm{kg}$ bodyweight and higher. Compared with the 12-week treatment duration, anaemia was more frequent in patients treated with SOF/RBV for 16 weeks (19.1\% vs 36.8\%) likely attributable to the higher proportion of patients with more advanced liver disease in the latter group.

SOF and RBV was generally safe and well tolerated in this real-world patient population that also included patients with advanced liver disease $(26.8 \%$ cirrhotic; prior decompensation

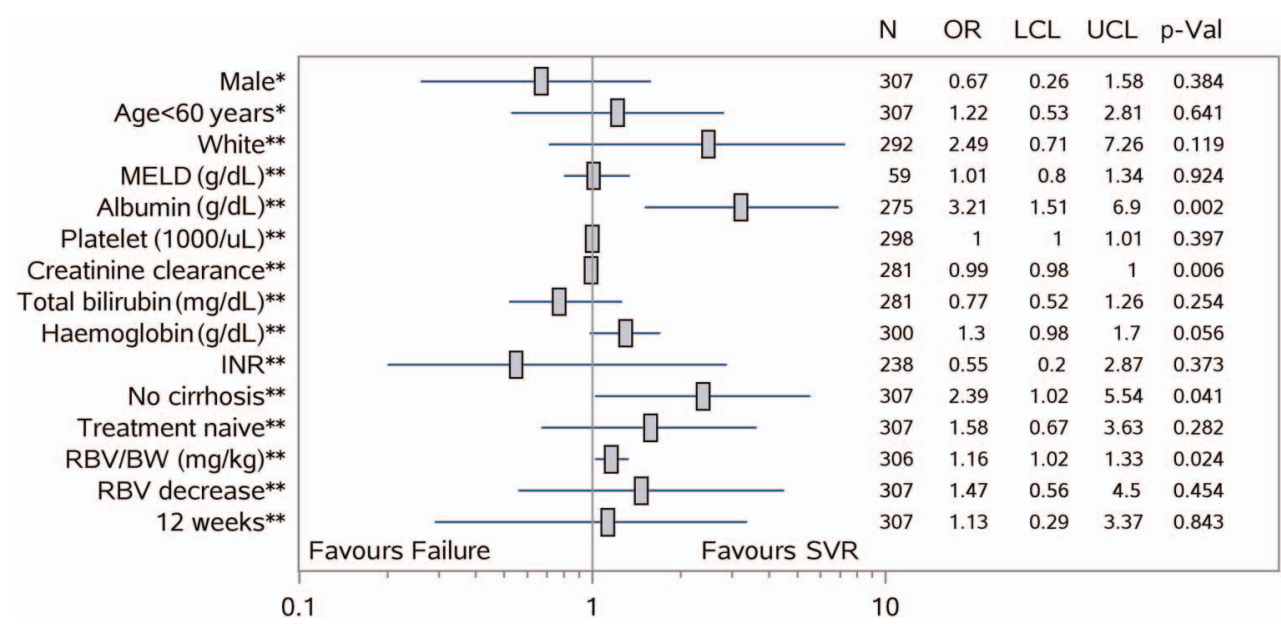

Figure 3 Predictors of sustained virological response (SVR) among genotype 2 (GT2)-infected patients who were treated with sofosbuvir (SOF) +ribavirin (RBV) and had an available virological outcome $(n=307)$. *Patients who discontinued due to adverse events or were lost to follow-up are excluded. $\mathrm{N}$, number observed; $\mathrm{OR}, 95 \% \mathrm{Cl}\left(\mathrm{CL}\right.$, confidence limit; $\mathrm{LCL}$, lower confidence limit; $\mathrm{UCL}$, upper confidence limit) and $p$ value. ${ }^{* *}$ Estimated with logistic regression with the predictor of interest, age and gender in the model. BW, bodyweight; INR, international normalised ratio; MELD, model for end-stage liver disease. 
Table 3 Adverse events (AEs) and management of anaemia

\begin{tabular}{|c|c|c|c|}
\hline Event & $\begin{array}{l}\text { Sofosbuvir (SOF) plus ribavirin } \\
\text { (RBV) for } 12 \text { weeks }(n=283) \\
\text { N patients }(\%)\end{array}$ & $\begin{array}{l}\text { SOF plus RBV for } 16 \\
\text { weeks }(n=38) \\
\text { N patients }(\%)\end{array}$ & $\begin{array}{l}\text { Total }(\mathrm{N}=321) \\
\mathrm{N} \text { patients }(\%)\end{array}$ \\
\hline AEs occurred in $>10 \%$ of patients & $234(82.7)$ & $34(89.5)$ & $268(83.5)$ \\
\hline Fatigue & $110(38.9)$ & $19(50.0)$ & $129(40.2)$ \\
\hline Anaemia & $54(19.1)$ & $14(36.8)$ & $68(21.2)$ \\
\hline Nausea & $51(18.0)$ & $5(13.2)$ & $56(17.5)$ \\
\hline Headache & $44(15.6)$ & $7(18.4)$ & $51(15.9)$ \\
\hline Insomnia & $41(14.5)$ & $6(15.8)$ & $47(14.6)$ \\
\hline Rash & 37 (13.1) & $7(18.4)$ & 44 (13.7) \\
\hline Flu-like symptoms & 36 (12.7) & $4(10.5)$ & 40 (12.5) \\
\hline Dyspnoea & $29(10.3)$ & $5(13.2)$ & $34(10.6)$ \\
\hline Back pain & $10(3.5)$ & $5(13.2)$ & $15(4.7)$ \\
\hline Serious AEs* & $11(3.9)$ & $4(10.5)$ & $15(4.7)$ \\
\hline \multicolumn{4}{|l|}{ Anaemia management } \\
\hline RBV dose reduction & $45(15.9)$ & $12(31.6)$ & $57(17.8)$ \\
\hline Erythropoetin use & $7(2.5)$ & $3(7.9)$ & $10(3.1)$ \\
\hline Blood transfusion & $7(2.5)$ & $1(2.6)$ & $8(2.5)$ \\
\hline RBV discontinuation & $1(0.4)$ & $0(0)$ & $1(0.3)$ \\
\hline
\end{tabular}

*Anaemia (2), abdominal pain (2), chest discomfort (1), chest pain (1), generalised oedema (2), multiorgan failure (1), bacterial peritonitis (2), gastroenteritis (1), upper respiratory tract infection (1), urinary tract infection (1), fall (1), fluid overload (1), hypovolaemia (1), hepatic encephalopathy (2), depression (1), chronic obstructive pulmonary disease (1) and stent placement (1). ^AE of anaemia reported by treatment provider, use of erythropoietin growth factors (such as eryphopoetin) or patient receiving a transfusion.

in $12.5 \%$ ). Most common AEs corresponded to those previously reported pivotal phase III studies. ${ }^{24}$ Treatment discontinuation due to AEs was infrequent $(2.5 \%)$ and most $(8 / 10)$ occurred in patients with advanced liver disease. Serious AEs were reported in $<5 \%$ of the patients. The somewhat higher rate compared with pivotal phase III studies might be attributable to the higher proportion of patients with (decompensated) cirrhosis in HCV-TARGET. All six deaths reported in this study were considered unrelated to the antiviral medication by the treating physicians. Cardiac arrest was reported as a cause of death after a road traffic accident and in a patient with decompensated cirrhosis. Underlying cardiac disease or intake of amiodarone recently reported to cause symptomatic bradycardia in combination with SOF was not reported in any of the two patients. Anaemia was frequent in this real-world population (21.2\%) and mostly managed with RBV dose reductions.

The strength of the present study is the large number of patients with GT2 infection, encompassing a high proportion of patients with cirrhosis (26.8\%), and exceeding respective patient numbers enrolled into any pivotal phase III trials. Central data collection and abstraction by trained personnel, as well as ongoing record monitoring, ensured a high quality of the abstracted data. In addition, the international design confers a high generalisability of the reported real-world data. As this clinical practice study has a non-randomised, observational design, differences between treatment groups should be interpreted cautiously. In particular, patients with cirrhosis treated for 16 weeks had more advanced liver disease than those treated for 12 weeks. Also, the low number of patients treated for 16 weeks limited, as in respective pivotal studies, again the comparison of different treatment durations, particularly in patients with liver cirrhosis. SVR12 has been shown to be associated with improvements in liver function parameters in patients with advanced liver disease. ${ }^{25} 26$ Follow-up in this study, however, might not have been long enough to detect statistically significant differences in this cohort. Also, information on the presence of resistance-associated variants in patients with virological failure is missing. It would have been interesting to investigate whether some of the treatment failures were attributable to GT2k/1b recombinant GTs; however, respective sequencing results were not available. ${ }^{27}$ As week 4 HCV RNA results were not routinely collected for patients treated with SOF and RBV, HCV RNA kinetics could not be investigated in this real-world cohort. Finally, some attrition bias caused by exclusion of 24 patients who did not complete treatment with SOF and RBV from the analysis cannot be excluded.

In summary, in this large, international cohort study, the all-oral combination of SOF and RBV was safe and effective for treatment of HCV GT2. While response rates in patients without cirrhosis were high and comparable with those reported in clinical trials, the presence of lower albumin levels and liver cirrhosis was associated with lower SVR12 rates. Larger, randomised trials in patients with cirrhosis are required to determine the benefit of extended treatment durations of SOF and RBV in this patient group. Recent studies showed that combined SOF and valpatasvir (ASTRAL-1, ASTRAL-2) yielded SVR12 rates of up to $100 \%$ in patients with GT2 and cirrhosis, so that the combination of SOF and a GT2-active NS5A inhibitor for 12 weeks may be preferable to SOF and RBV for more than 12 weeks in patients with GT2 and liver cirrhosis. ${ }^{28} 29$

\section{Author affiliations}

${ }^{1} \mathrm{JW}$ Goethe University Hospital, Frankfurt am Main, Germany

${ }^{2}$ University of Florida, Gainesville, Florida, USA

${ }^{3}$ Saint Louis University School of Medicine, St. Louis, Missouri, USA

${ }^{4}$ University of Pennsylvania, Philadelphia, Pennsylvania, USA

${ }^{5}$ University of California, San Diego, San Diego, California, USA

${ }^{6}$ Yale University of School of Medicine, New Haven, Connecticut, USA

${ }^{7}$ University of North Carolina, Chapel Hill, North Carolina, USA

${ }^{8}$ Scripps Clinic, La Jolla, California, USA

${ }^{9}$ Research Specialists of Texas, Houston, Texas, USA

${ }^{10}$ Liver Wellness Clinic, Little Rock, Arkansas, USA

${ }^{11}$ Johns Hopkins University, Baltimore, Maryland, USA

Contributors All authors had access to the data and approved the final version of the manuscript. 
Funding HCV-TARGET is an investigator-initiated study jointly sponsored by The University of Florida, Gainesville, FL (PI: Nelson), and The University of North Carolina at Chapel Hill, Chapel Hill, NC (PI: Fried). It was funded in part by AbbVie, BristolMyers Squibb, Gilead, Janssen, Kadmon, Merck and Vertex. Funded in part by CTSA UF UL1TR000064. Dr Fried was funded in part by National Institutes of Health (NIH) Mid-Career Mentoring Award K24 DK066144. Dr Sherman was funded in part by NIH K24 DK070528.

Competing interests TMW: Consultancies/speaker for Abbvie, Bristol-Myers Squibb, Gilead, Janssen. DRN: Grant funding from AbbVie, Gilead, BMS, Janssen, Merck, GSK. GM: Grant funding from AbbVie, BMs, Gilead, Merck, Janssen, Vertex, Idenix, Conatus and Salix. ADB: Grant funding from Gilead, AbbVie and BMS. Consultant for Gilead, AbbVie and BMS. Expert testimony/advisory board from Gilead, AbbVie and BMS. RKR: Grant funding from AbbVie, Merck, Gilead, Janssen, Vertex (Money paid to Pennsylvania). Expert testimony/advisory board from AbbVie Merck, Gilead, BMS, Janssen. AK: Grant funding from Gilead. JKL: Grant funding from BMS, Gilead, Janssen, Hologic (All to institution). Consultant to BMS, Gilead, Janssen. JD: Grant funding and consulting from BMS. PP: Grant funding from Gilead, BMS, AbbVie, Merck, Janssen. Consultant for Gilead, BMS, AbbVie, Merck and Janssen. Sponsored lectures/Honoraria from Gilead, BMS, AbbVie, Janssen. JSG: Grant funding from Merck and Gilead. Sponsored lectures/Honoraria from Gilead Speakers Bureau. LMF: Grant funding and consulting from Gilead, AbbVie, Janssen and Merck. SA: Grant funding from Gilead, AbbVie and Merck. Consultant for Gilead, AbbVie, Merck and Janssen. Sponsored Lectures/Honoraria from Gilead and AbbVie. MSS: Grant funding from AbbVie, BMS, Gilead, Janssen and Merck. (Paid to Johns Hopkins University, NIH/NDA K24DA034621). Consultant for AbbVie, CoCrystal Pharma, Gilead, Janssen, Merck and Trek. MV: No Disclosures. LA: No Disclosures. MWF: Grant funding from Merck, Janssen, Gilead, BMS, AbbVie and research grants from NIH. Consultant for Merck, AbbVie, Gilead and BMS. SZ: Consultant for AbbVie, BMS, Gilead, Janssen and Merck. Sponsored Lectures/ Honoraria from AbbVie, BMS, Gilead, Janssen and Merck.

Patient consent Obtained.

Ethics approval The protocol was approved by an independent ethics committee or a central institutional review board (IRB) according to country-specific and site-specific regulations and UF IRB.

Provenance and peer review Not commissioned; externally peer reviewed.

Data sharing statement Data collection. Demographic and clinical information, laboratory tests and technical exams were performed according to the site's standards. Data were collected within a centralised database using standardised source data abstraction as described previously. The database was managed using Research Electronic Data Capture (REDCap) tools hosted at the University of North Carolina, Chapel Hill. To optimise data quality, a trained centralised Chart Data Abstraction Team at the Clinical Coordinating Center at the University of Florida was established to enter de-identified copies of source data, that is, clinical records that were provided by participating sites. Special effort was made to explore reasons for premature discontinuations and to investigate the reasons for missing data and to minimise loss to follow-up. Independent data monitors reviewed data entries for completeness and accuracy.

Open Access This is an Open Access article distributed in accordance with the Creative Commons Attribution Non Commercial (CC BY-NC 4.0) license, which permits others to distribute, remix, adapt, build upon this work non-commercially, and license their derivative works on different terms, provided the original work is properly cited and the use is non-commercial. See: http://creativecommons.org/ licenses/by-nc/4.0/

\section{REFERENCES}

1 Thomas DL. Global control of hepatitis C: where challenge meets opportunity. Nat Med 2013;19:850-8.

2 Westbrook RH, Dusheiko G. Natural history of hepatitis C. J Hepatol 2014;61 (1 Suppl):S58-68.

3 van der Meer AJ, Wedemeyer $\mathrm{H}$, Feld JJ, et al. Life expectancy in patients with chronic HCV infection and cirrhosis compared with a general population. JAMA 2014;312:1927-8.

4 van der Meer AJ, Veldt BJ, Feld JJ, et al. Association between sustained virological response and all-cause mortality among patients with chronic hepatitis $C$ and advanced hepatic fibrosis. JAMA 2012;308:2584-93.
5 Gower E, Estes C, Blach S, et al. Global epidemiology and genotype distribution of the hepatitis C virus infection. J Hepatol 2014;61(1 Suppl):S45-57.

6 Messina JP, Humphreys I, Flaxman A, et al. Global distribution and prevalence of hepatitis C virus genotypes. Hepatology 2015;61:77-87.

7 Andriulli A, Mangia A, lacobellis A, et al. Meta-analysis: the outcome of anti-viral therapy in HCV genotype 2 and genotype 3 infected patients with chronic hepatitis. Aliment Pharmacol Ther 2008;28:397-404.

8 Welzel TM, Dultz G, Zeuzem S. Interferon-free antiviral combination therapies without nucleosidic polymerase inhibitors. J Hepatol 2014;61(1 Suppl):S98-S107.

9 Kumar S, Jacobson IM. Antiviral therapy with nucleotide polymerase inhibitors for chronic hepatitis C. J Hepatol 2014;61(1 Suppl):S91-7.

10 Sovaldi (sofosbuvir) prescribing information. Foster City, CA: Gilead Sciences, 2014 (package insert). (http://www.gilead.com/ /media/Files/pdfs/medicines/liver-disease/ sovaldi/sovaldi_pi.pdf).

11 Lawitz E, Mangia A, Wyles D, et al. Sofosbuvir for previously untreated chronic hepatitis C infection. N Engl J Med 2013;368:1878-87.

12 Jacobson IM, Gordon SC, Kowdley KV, et al., POSITRON Study; FUSION Study. Sofosbuvir for hepatitis C genotype 2 or 3 in patients without treatment options. N Engl J Med 2013;368:1867-77.

13 Zeuzem S, Dusheiko GM, Salupere R, et al., VALENCE Investigators. Sofosbuvir and ribavirin in HCV genotypes 2 and 3. N Engl J Med 2014:370:1993-2001.

14 AASLD/IDSA HCV Guidance Panel. Hepatitis C Guidance: AASLD-IDSA Recommendations for Testing, Managing, and Treating Adults Infected With Hepatitis C Virus. Hepatology 2015;62:932-54.

15 Myers RP, Shah H, Burak KW, et al. Re: RP Myers, H Shah, KW Burak, et al. An update on the management of chronic hepatitis C: 2015 Consensus guidelines from the Canadian Association for the Study of the Liver. Can J Gastroenterol Hepatol 2015;29:19-34.

16 European Association for Study of Liver. EASL Clinical Practice Guidelines: management of hepatitis C virus infection. J Hepatol 2014;60:392-420.

17 Sarrazin C, Berg T, Buggisch P, et al. [S3 guideline hepatitis C addendum]. Z Gastroenterol 2015:53:320-34.

18 Dieterich D, Bacon B, Flamm S, et al. P0775: Final evaluation of 955 HCV patients treated with 12 week regimens containing sofosbuvir $+/-$ simeprevir in the trio network: Academic and community treatment of a real-world, heterogeneous population. J Hepatol 2015;62:S621

19 Backus LI, Belperio PS, Shahoumian TA, et al. Effectiveness of sofosbuvir-based regimens in genotype 1 and 2 hepatitis C virus infection in 4026 U.S. Veterans. Aliment Pharmacol Ther 2015:42:559-73.

20 http://www.hcvtarget.org (accessed on 01/09/2016)

21 Sulkowski MS, Vargas HE, Di Bisceglie AM, et al., HCV-TARGET Study Group. Effectiveness of Simeprevir Plus Sofosbuvir, With or Without Ribavirin, in Real-World Patients With HCV Genotype 1 Infection. Gastroenterology 2016:150:419-29.

22 Harris PA, Taylor R, Thielke R, et al. Research electronic data capture (REDCap) - a metadata-driven methodology and workflow process for providing translational research informatics support. J Biomed Inform 2009;42:377-81.

23 Foster GR, Pianko S, Brown A, et al., BOSON Study Group. Efficacy of Sofosbuvir Plus Ribavirin With or Without Peginterferon-Alfa in Patients with Hepatitis C Virus Genotype 3 Infection and Treatment-Experienced Patients With Cirrhosis and Hepatitis C Virus Genotype 2 Infection. Gastroenterology 2015;149:1462-70.

24 Reddy KR, Bourlière $M$, Sulkowski $M$, et al. Ledipasvir and sofosbuvir in patients with genotype 1 hepatitis $C$ virus infection and compensated cirrhosis: An integrated safety and efficacy analysis. Hepatology 2015;62:79-86.

25 Deterding K, Höner Zu Siederdissen C, Port K, et al. Improvement of liver function parameters in advanced HCV-associated liver cirrhosis by IFN-free antiviral therapies. Aliment Pharmacol Ther 2015 Oct:42:889-901.

26 Charlton M, Everson GT, Flamm SL, et al., SOLAR-1 Investigators. Ledipasvir and Sofosbuvir Plus Ribavirin for Treatment of HCV Infection in Patients With Advanced Liver Disease. Gastroenterology 2015;149:649-59.

27 Karchava M, Waldenström J, Parker $\mathrm{M}$, et al. High incidence of the hepatitis C virus recombinant $2 \mathrm{k} / 1 \mathrm{~b}$ in Georgia: Recommendations for testing and treatment. Hepatol Res 2015;45:1292-8. Dec.

28 Feld JJ, Jacobson IM, Hézode C, et al., ASTRAL-1 Investigators. Sofosbuvir and Velpatasvi for HCV Genotype 1, 2, 4, 5, and 6 Infection. N Engl J Med 2015;373:2599-607.

29 Foster GR, Afdhal N, Roberts SK, et al., ASTRAL-2 Investigators; ASTRAL-3 Investigators. Sofosbuvir and Velpatasvir for HCV Genotype 2 and 3 Infection. N Engl J Med 2015;373:2608-17. 